\title{
Traffic Light Control Using Image Processing
}

\author{
Jaya Singh ${ }^{1}$, S. K. Singh ${ }^{2}$ \\ ${ }^{1}$ MTech(C.S), Dr. A.P.J. Abdul Kalam Technical University, Vishveshwarya Institute of Engineering Technology, \\ Ghaziabad-Bulandshahr G.T. Road, P.O.-NH-91 G, Dadri Main Rd, Greater Noida, U.P. 203207
}

${ }^{2}$ Associate Professor (C.S. Department) Dr. A.P.J. Abdul Kalam Technical University, Vishveshwarya Institute of Engineering Technology, Ghaziabad-Bulandshahr G.T. Road, P.O.-NH-91 G, Dadri Main Rd, Greater Noida, U.P. 203207

\begin{abstract}
As we know that, the populaces is developing step by step, and as the populace is developing the quantity of vehicles out and about are likewise developing. As now days, number of vehicles out and about are bigger than the space on street. The traffic issues are expanding now days in view of the developing number of vehicles and the constrained assets gave. The first and the least difficult route is to utilize clock for every path for controlling a traffic light .And another route is to utilize electronic sensors to distinguish vehicles, and produce signal.. Also, in this we proposed a framework that controls the traffic light by image processing. This framework will recognize vehicles through images. A camera will be introduced close by the traffic light and after that it will catch image arrangements. The image grouping will then be investigated utilizing digital image processing for vehicle location, and as per traffic conditions out and about, traffic light will be controlled.
\end{abstract}

Keywords: RGB, Grayscale, Image Acquisition, Morphological operations.

\section{Introduction}

In later and future years, traffic clog has turned into a noteworthy issue. Traffic clog and sticks are one of the fundamental purposes behind expanding transportation costs because of the squandered time and additional fuel.

\section{-Building new streets and paths are simply unrealistic any more, however building insight into the streets what's more, paths - with cutting edge innovation-is surely conceivable..-}

Current traffic control procedures including magnetic loop detectors covered in the street, infra-red and radar sensors as an afterthought give restricted traffic data and require separate frameworks for traffic numbering and for traffic observation magnetic loop detectors have been the most utilized advances, yet their establishment and upkeep are awkward and might get to be contradictory with future base.

Fumble and traffic blockage results in long holding up and loss of fuel and cash. The observing and controlling of city traffic is turning into a noteworthy issue in numerous nations. With expansion in number of vehicles out and about, the

Traffic Monitoring Authority needs to discover new strategies for succeeding such an issue. The assets gave by current foundations are constrained and the quantity of street clients always increments, clever control of traffic will turn into a vital issue later on. Traffic clog might come about because of substantial traffic at an intersection. There are such a variety of traffic administration methods accessible to maintain a strategic distance from clog on street. In any case, no method is immaculate without anyone else's input as the ongoing circumstances are for the most part persistently changing and the framework needs to adjust to change in the ceaselessly changing circumstances and developing populace We have made an endeavor to give some traffic administration procedure which is self-changing in nature, in order to fit into ceaselessly changing ongoing traffic situations. In this framework time is relegated to every path of traffic light, as per the traffic thickness out and about.

\section{Existing Systems}

Pezhman Niksaz et. al. [1] propose a framework that gauges the size of traffic in thruways by utilizing image preparing has been proposed and thus a message is appeared to educate the number of vehicles in expressway. This task has been executed by utilizing the Matlab software and it means to avert substantial traffic in expressways. In addition, for executing this venture taking after steps must be considered: 1) image acquisition 2) RGB to grayscale transformation 3) image enhancement and 4) morphological operations. At to start with, film of thruway is caught by a camera has been introduced in expressway. At that point, the film comes as continuous casings and every edge is contrasted and the primary casing. After that, the quantity of vehicle in roadways is determined. Toward the end, if the quantity of vehicle is more than a limit, a message is appeared to advise the traffic status. By this message we can foresee the need to diminish the measure of traffic conveyed. Tests demonstrate that the calculation will work legitimately.

In this specific research paper they have utilized video camera. Camera is shooting video and the video is then changed over to succession of images by taking previews. This is entirely troublesome as we are nothing to do with video scope. So we maintained a strategic distance from this strategy and chose to utilize a basic camera as it were.

Vikramaditya Dangi, Amol Parab, Kshitij Pawar and S.S Rathod [2] propose the best approach to actualize a keen traffic controller utilizing real time image processing. The image groupings from a camera are dissected utilizing different edge detection and item checking techniques to acquire the most effective system. In this way, the quantity of vehicles at the crossing point is assessed and traffic is effectively oversaw. The paper likewise proposes to actualize an ongoing crisis vehicle location framework. In the event 


\section{International Journal of Science and Research (IJSR) \\ ISSN (Online): 2319-7064}

Index Copernicus Value (2013): 6.14 | Impact Factor (2014): 5.611

that a crisis vehicle is distinguished, the path is given need over all the others.

The key purpose of this paper is the strategy which is utilized for edge detection. The authors have given the examination of different edge detection procedures and reason that canny edge detection is the best technique for edge detection. Along these lines we are utilizing canny edge detection.

Niranjan Bhattacharayya, Akshay Kumar Singh, Siddhant Kapil, Harshul Jain, Abhishek Jain[3] proposes a system that tells that image processing is the best technique for controlling traffic light. In this, they proposes an algorithm that uses color detection, shape detection and coordinate analysis to detect and analyse the traffic and a response system for a UGV(Unmanned Ground Vehicle).

Omkar Ramdas Gaikwad, Anil Vishwasrao, Prof. Kanchan Pujari, Tejas Talathi [4] proposes a system that also uses image processing to control the traffic. In this paper they uses the technique in which we draw three green lines on each signal at arbitrary distances which represent: low, medium, and high. In this green color is specifically used because the researcher assume that the vehicles of green color are less in frequency, so it is easy to differentiate between the lines drawn and vehicles.

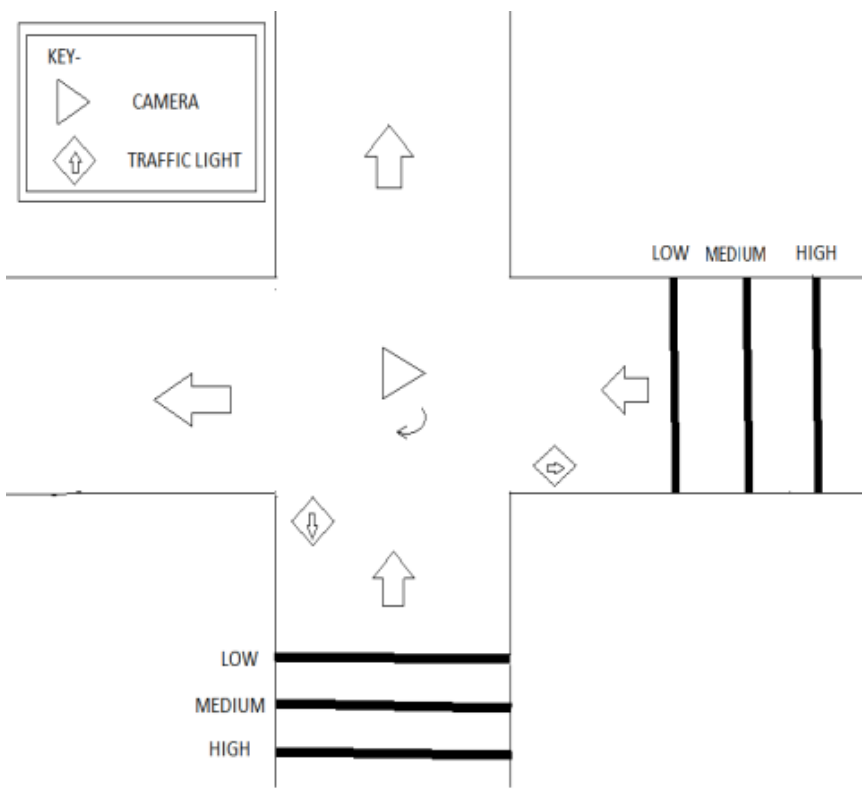

Figure 1

A camera is placed on each signal such that it focuses on the green lines. These green lines determine the traffic quantity by counting the pixels. If all the green lines are visible at a signal it means the traffic is very low and then we will provide the less time to that signal suppose 20 seconds. Now if two lines are visible it shows the traffic is medium and for this we will assign some more time to that signal as previous one suppose 35 seconds. And if no line is visible, it shows that traffic is too heavy such that we should assign much more time than the last one suppose 50 seconds. Therefore the optimization of switching between traffic light increases and traffic congestion reduces.

\section{Implementation of the Existing System}

Omkar Ramdas Gaikwad, Anil Vishwasrao, Prof. Kanchan Pujari, Tejas Talathi [4] implemented the system in following way:

Step1-

In this step the image is received via camera in RGB format, firstly this is to be converted into grayscale .so we use MATLAB for this purpose.

\section{A) RGB to gray conversion-}

We can model a color image as the band of three monochrome image data, the three data band represents the different color. In the digital image data the brightness information is stored for each spectral band. Whenever the image is displayed there brightness information is given on the screen by picture element that emits light energy to that particular color.

\section{B) Green Color Subtraction}

For measuring pixel count we should implement green color subtraction.

\section{C) Threshold -}

The yield image of non-maximum suppression stage might comprise of broken edge shapes, single edge points which add to noise. This can be disposed of by thresholding with hysteresis. Two limits are considered for hysteresis, one high limit other low edge. In the event that any edge response is over a high limit, those pixels constitute definite edge yield of the indicator for a specific scale. Individual feeble response for the most part compare to noise, yet in the event that these points are associated with any of the pixels with high limit, they are more prone to be real edges in the image. Such associated pixels are dealt with as edge pixels if their reaction is over a low edge. To get slender edges two limits (high edge (TH) and low edge (TL)) are utilized. In the event that the slope of the edge pixel is over the $\mathrm{TH}$, it is considered as an edge pixel. On the off chance that the inclination of the edge pixel is underneath TL then it is unequivocally set to zero. In the event that the slope is between these two, at that point it is set to zero unless there is a way from this pixel to a pixel with an inclination above $\mathrm{TH}$ ; the way must be completely through pixels with slopes of in any event TL.

\section{D) Black and white conversion-}

Now the image is converted into black and white image so that we can use it in future.

Step2 -

As the image is in black and white form. The green strip represents the white color and the background is of black color. In this we have measured green area of an image received.in this the author consider the area say 10,000 pixels and then each strip must be having 3300 pixels approx. by using this pixel information we can define the three traffic conditions which were: low, medium, and high. If the green count is between $7000-10,000$, then it is said to be low traffic 


\section{International Journal of Science and Research (IJSR) \\ ISSN (Online): 2319-7064 \\ Index Copernicus Value (2013): 6.14 | Impact Factor (2014): 5.611}

and accordingly we provide the time interval suppose 20 seconds. If the pixel count is between 3500-7000 then it is said to be medium traffic and accordingly the time is allotted suppose 35 seconds. Now if the pixel count is more than 0 then it is said to be having heavy traffic and accordingly time is allotted suppose 45 seconds. And if the pixel count is 10000 then there is no traffic so no time or minimum time is allotted to it.

Step3-

Thus according to pixel count a code is generated in MATLAB which is to be sent to controller to allocate timer.

For example-

High traffic- 45 seconds

Medium traffic-35 seconds

Low traffic-25 seconds

No traffic- default (minimum)

Step 4-

In this step the microcontroller based hardware unit receives a code through serial port. So, depending upon the code received, the time is allocated by the controller to the traffic timer of each signal.

\section{Drawback of the Existing System}

Omkar Ramdas Gaikwad, Anil Vishwasrao, Prof. Kanchan Pujari, Tejas Talathi [4] proposed a system in which we used the green color strips to count the density of traffic. In this system they assumed that green color vehicles are rarely used. But what if the vehicles at a signal are mostly of green color, than we are not able to determine the traffic density at a signal.

\section{Conclusion and Future Work}

In this paper we studied the existing system that is using image processing for traffic light controlling. In this we studied that we are using camera for capturing image and then using green strips to determine the traffic count. But as given above the drawback of the existing system. In future try to overcome the drawback of the existing system.

\section{References}

[1] Pezhman Niksaz, Science \&Research Branch, Azad University of Yazd, Iran, "Automatic Traffic Estimation Using Image Processing ",2012 International Conference on Image, Vision and Computing.

[2] Vikramaditya Dangi, Amol Parab, Kshitij Pawar \& S.S Rathod "Image Processing Based Intelligent Traffic Controller", Undergraduate Academic Research Journal (UARJ), ISSN: 2278 - 1129, Volume-1, Issue-1, 2012.

[3] Niranjan Bhattacharayya, Akshay Kumar Singh, Siddhant Kapil, Harshul Jain, Abhishek Jain "Traffic Light Solution for U.G.V. Using Digital Image Processing”, International Journal of Soft Computing and Engineering (IJSCE) ISSN: 2231-2307, Volume-4, Issue-ICCIN2K14, March 2014.
[4] Omkar Ramdas Gaikwad, Anil Vishwasrao, Prof. Kanchan Pujari, Tejas Talathi, "Image Processing Based Traffic Light Control”, International Journal of Science, Engineering and Technology Research (IJSETR) Volume 3, Issue 4, April 2014.

\section{Author Profile}

Jaya Singh is an M.Tech student of VIET, Dr. A.P.J. Abdul Kalam Technical University. Her specialization is in Computer Science.

S. K. Singh is an Associate Professor (Computer Science Department) in VIET, Dr. A.P.J. Abdul Kalam Technical University. 